\title{
ADP Ribosylation
}

National Cancer Institute

\section{Source}

National Cancer Institute. ADP Ribosylation. NCI Thesaurus. Code C129423.

The transfer of ADP-ribose to amino acids within proteins or to the amino group at N2 of a 2'-deoxyguanosine residue in double-stranded DNA. 